\title{
Filamento espiral perinuclear de Hortega-Górriz na fibra muscular estriada humana e no myocardio
}

\author{
Nota prévia de M. DE FREITAS AMORIM, alumno interno
}

Applicados a principio quasi exclusivamente nas investigações sob) o systema nervoşo, o uso dos methodos de impregnação metallica nas pesquizas sobre os outros tecidos produziu resultados os 11:ais lisongeiros. Assim é que o estudo da estructura intima do tecido muscular tambem soube auferir grandes vantagens dessa generalização, muitos tendo sido os factos novos verificados a custa desses poderosos meios de investigação histologica.

Ainda recentemente o emprego de uma das varias modificações do methodo classico da prata reduzida de Cajal, o methodo uranoargentico, ideado pelo proprio CAJAL com o fim de facilitar a demonstração do apparelhe endocellular de Golgi, veiu enriquecer o numero de nossos conhecimentos nesse sentido com a verificação cie uma nova particularidade de estructura da fibra muscular. Quetemo-nos referir ao filamento espiral perinuclear, objecto da presente nota.

Descripto pela primeira vez em 1913 por DEL RIO-HORTEGA (1) nas filro-cellulas musculares do utero, estomago, intestino e bexiga de animaes jovens, a existencia do filamento em questão foi confirmada somente em Março do corrente anno por M. GORRIZ (2), que, fazendo $i_{1}$ :estigações com o referido methodo sobre os musculos esqueletticos ce batracios e de alguns mammiferos, surprehendeu a sua presença tambem nas fibras musculares estriadas. A's varias questões impli-

(1) P. DEL RIO-HORTEGA. "Investigations sur le tisu musculaire lisse" Tralıajos del Laboratorio de investigaciones biologicas. Madrid. Tomo XI. 1913.

(2) M. GóRRIZ. "Sobre um filamento espiral perinuclear de las fibras musculares estriadas" Trabajos etc. Tomo XIX. Março de 1922. 
citas á existencia desse organito nuclear, como sejam a da sua situação (si intra ou extra-nuclear) e a da sua funcção, GORRIZ soube responder de uma maneira mais ou menos definitiva, fixando sua situação perinuclear e ao mesmo tempo demonstrando experimentaimente o seu papel passivo na contraç̧ão, durante a qual o filamento espiral, distendido pelo augmento das dimensões transversaes do nucleo, impediria que esse augmento transgredisse um certo limite.

Quanto á questão igualmente complexa attinente á intima natlireza chimica do dicto filamento, ella ainda permanece obscura, não cistante os trabalhos de HORTEGA e GORRIZ terem demonstrado de modo irrefutavel sua natureza distincta da chromatina, que nos batrácios (v. GEHUCHTEN) (1) affiectaria tambem uma disposição espiral semelhante. Aliás, o filamento espiral chromatinico descripto por v. GEHUCHTEN só encontrou confirmação por parte de MUNCH (2); HEIDERICH e HENNEBERG não falam sobre essa disposição e FORSTER ( $\left.{ }^{3}\right)$, en um minucioso estudo sobre os varios aspectos morphologicos apresentados pelo nucleo das cellulas musculares lisas e dos segmentos cardiacos, nega radicalmente a espiral nucleinica, mostrando como v. GEHUCHTEN dera uma interpreta(ఓ́) erronea á forma em espiral impressa aos nucleos pelo movimento de torsão, que toda a fibra muscular soffreria durante a contracção. Além disso, como faz notar GORRIZ, contrastando com a affinidade nulla ou quasi nulla que a nucleina apresenta pela prata colloidal, a demonstracção do filamento argentophilo do nucleo só se consegue com a prata reduzida, que, em determinadas circumstancias (previa fixação no liquido formol-uranico, integridade da chromatina nuclar), o impregna electivamente.

Aconselhados pelo professor BOVERO, a effectuar pesquizas com o fim de demonstrar a possivel existencia do filamento perinuclear de Hortega-Górriz nos segmentos da musculatura cardiaca, emprehendemos nesse sentido varias tentativas, ao mesmo tempo que tratavamos de verificar tambem a existencia e as particularidades do dicto filamento na fibra muscular estriada humana, ainda não explorada nesse particular.

Nos musculos voluntarios do nomem as nossas tentativas foram deste logo coroadas do mais pleno exito, como tivemos occasião de communicar á Sociedade de Medicina e Cirurgia de S. Paulo na

(1) V. GEHUCHTEN. "Les noyaux des cellules musculaires strieés de la grenouille adulte" Anatomischer Anzeiger, Bd. 4. n. 2. s. 52-64, 24-1-1889.

(2) MUNCH. K. "Ueber Nucleinspiralen im Kern glatten Muskelzellen". Arch. f. mikrosk. Anat. Bd. 62, 1903.

(3) E. FORSTER. "Die Kontraktion der glatten Muskelzellen und der Herzmuskelzellen" Anatomischer Anzeiger, Bd. 25; n. 14-15; s. 338-355; 16-9-1904. 
sessão de 1 de Julho deste anno. A mesma facilidade, porem, nãn encontramos quanto á demonstração do filamento nos nucleos do syncicio cardiaco, demonstração essa que até aquella epoca não haviamos conseguido, embora tivessemos tentado varias vezes e em differentes animaes. Mas, continuando depois disso as nossas investigaçôes. conseguimos afinal apoz muitas outras tentativas improficuas obter a impregnação do apparelho filamentar nos segmentos io myocardio de cohaya, constituindo esse achado o objecto de uma nova communicaçãc á referida Socicdade em 15 do mesmo mez de Julho.

\section{Fılamento perinuclear na fibra estriada humana}

As observações foram feitas em fragmentos de musculos largos de homem adulto retirados durante uma operação de hernia inguinal, e de musculos longos excisados por biopsia em um individuo portador da molestia de Thomsen (Myotoria congenita). A nota presente se refere exclusivamente ás disposições encontradas nos musculos normaes, reservando-nos para publicar mais tarde, em collaboração com o nosso collega F. CINTRA DO PRADO, os resultados das nossas pesquizas sobre o comportamento do filamento argentophilo perinuclear na Myotonia congenita.

Nas suas linhas geraes a technica empregada foi a seguinte: fixação immediata de pequenos pedaços em formoli a $15 \%$ contendo $1 \%$ de nitrato de uranio (depois de 10 horas de fixação já se obtem ás vezes a impregnação do filamento); immersão em um banho de nitráto de prata a $1,5 \%$ durante 48 horas; reducção pelo acido pyrogailico ou hydroquinona; inclusão em parafina.

Nas preparações felizes vê-se, muitas vezes com a mais perfeita nitidez, um delicardo filamento energicamente impregnado em inegro pela prata reduzida, cujas voltas circumscrevem o nucleo to to á maneira de uma espiral (fig. 1). Nas fibras fixadas em repouso, o filamento espiral enlaça suavemente o nucleo, conservando-lhe o contorno ellipsoide, ao passo que nas fibras fixadas em contração, o nucleo se mostra estrangulado em varios pontos, como si o filamento perinuclear o amarrasse solidamente. As voltas descriptas pelo filamento en tcrno do nucleo são sempre mais ou menos obliquas em relação ao maior eixo nuclear e conservam uma espessura regularmente constant: salvo nos pólos nucleares, onde a espiral termina por uma extremidade gradualmente afilada e pallida.

GORRIZ, provocando a contracção tetanica de um musculo por excitação com corrente faradica durante 30 minutos e íixando-o nessas condições por meio de iniecções intersticiaes do liquido formol-uranico, obteve imagens do filamento espiral, em que as alsas 
se apresentavam perfeitamente rectilineas, como si o augmento do diametro equatorial do nucleo durante a contracção tivesse retezado violentamente as voltas do filamento.

No que diz respeito ao aspecto das alsas filamentares verificadas nas conciçōes haỉitaes de observação, isto é, nas fibras cuja contracção é provocada pelo proprio liquido fixador agindo como excitante, as noşsas observações confirmam fundamentalmente as de GORRIZ. Com effeito, pudemos notar que as alsas do apparelho fiiamentar desenham um trajecto irregular, ás vezes apenas colleante de maneira a quuasi apparentar uma direç̧ão rectilinea, outras manifestamente tortuoso, descrevendo linhas sinuosas, ou mesmo quebrando-se em angulos mais ou menos pronunciados, em ziguezague.

Do exame cirecto dessas irregularidades do trajecto filamentar e secundacios pela observação de GORRIZ acima citada, acinamos licito pensar que o estado de distensão ou de relachamento las esp:raels indica um grau correspondente de contracção ou de repouso da fibra musciiar, ou, em outras palavras, - maior ou menor grau de distensão do tilamento perinuclear .orstitue um meio indi:ecto que nos permittiria quasi sempre avaliar do estado de con .i acção da fibra niuscular em $\cdots$ ame.

Nos nossos jepiados os nucleos, slic alsas fitimentare; se mostram reiativamente distendidas, apresentam sempre am perfil fran! ado, que lhes dá uma forma ellipsoide, em sacca-rolhas. Segundo a ,opinịa de GORRIZ, esse aspecto seria devido ao facto que durante a : contrecção a massa nuclear, tendendo a espandir-se no sentido transversal, ao se insinuar entre as voltas do filamento, "formaria hernia" Aliaz, o perfil franjado ou a forma serpentina do nucleo se oḅserva tambem nas preparações effecttuadas segundio os processos technicos mais usuaes, tanto na fibro-lcellula como na fibra estriada e nos segmentos cardiacos. (Vejam-se a este respeito as imagens que figuram nos tratados ou nas memorias ide G. SCHWAIBE (1868), ARNOLD (1871), RENAUT (1888), SCHAFFER (1899), v. IJENHOSSEK (1899), STOHR (1901), FORSTER (1904), MC. GILL (1908), FUSARI (1909), HEIDENHAIN (1911), POLICARD (1922), etc.). $\mathrm{E}$, ás vezes, o aspecto serpentino que o nucleo assume com as coloraçôes communs é tão typico, que não seria de toto impossivel com o exame meticuloso do perfil nuclear prevêr a existencia do łilamento ferinuclear contensor do nucleo.

Segundo as nossas observações, o numero de espiraes varia entre 3 e 10. Curioso é o facto do numero minimo de espiraes se observar quasi sempre em nucleos seccionados tangencialmente ás margens. Comprehende-se logo, porém), a razão. de sler deste farctio lembrando-nos que as faces nucleares, offerecendo uma maior superficie do que as margens, é sobre ellas naturalmente que deverá 
terminar com mais frequencia o filamento, e, nesse caso, à extremidade terminal do filamento não sendo vista pela margem nuclear, conta-se uma espiral de menos.

Sob o ponto de vista do numero de espiraes, GORRIZ descreveu duas modalidades do filamento nuclear: o denso ou apertado, que seria constituido por 10 a 20 espiraes, e o frouxo contendo apanas 4 ou 6. Dentro desses dois typos o A. distingue ainda o subtypo com= pleto ou polar, em que o filamento circumscreve o nucleo inteiro indo de polo a polo nuclear, e o incompleto ou equatorial, em que o filamento pouco desenvolvido envolveria apenas a parte central do nucleo.

Como se vê, talvez devido ao numero reduzido de nossas observações em relação ás de GORRIZ, até agora ainda não pudemos coiliirmar na fibra estriada humana essa classificação, porquanto nã verificamos nem uma só vez a presença de nucleos que pudessen: ser enquadrados no 1.' typo de GORRIZ, aliaz considerados por esse A. como constituindo a terça parte dos nucleos cujo apparelho filamentar se impregnou. Alem idisiso, a grande maioria dos filamentos perinucleares observados por nós no homem pertencem ao typo com1.jeto ou polar Só logramos notar a disposição equatorial do filamento referida por GORRIZ em nucleos apanhados tangencialmente ás margens, e mesmo nestes raros casos, pelo que dissemos anteriormente, não podemos affirmar si se trata de verdadeiros filamentos do typo equatorial ou de simples imagens equatoriaes de filamentos perfeitamente polares, cujas extremidades teriam terminado sobre as faces nucleares.

Quando o córte attinge tangencialmente a margem nuclear e sob v'm plano obliquo ao eixo transversal, têm-se uma interessante imagem do filamento, que neste caso apparenta ser constituido por varios anneis encaixados uns nos outros como os élos de uma corrente.

Nos casos em que o nucleo é seccionado longitudinalmente a imagem do filamento é incompleta, vendo-se apenas o perfil ellipsoide do nucleo angustiado nos pontos em que as alsas do filamento foram seccionadas.

Uma ou outra vez nossa attenção se fixou sobre nucleos com a n:embrana nuclear apparentemente integra e apresentando, entretanito, partes do filamento argentophilo (ora uma das extremidades, ora as alsas centraes) como que separadas da superficie nuclear (fig. 2). Outras vezes. porem, pudemos observar disposiçôes inversas do apparelho filamentar, em que uma ou varias alsas da espiral pareciam residir dentro do nucleo, como si estivessem intimamente adossadas. á face interna da membrana nuclear, que neste caso parecia vir tomar inserção sobre ellas. Estes dois factos, apparentemente contra- 
तíctorios no que diz respeito á situação do filamento argentophilo, ncs levaram a não abraçar "in totum" a opinião de GORRIZ, segando o qual o filamento se disporia "por fóra da membrana nucicar"

Parece-nos, antes, quẹ o filamento de Hortega-Górriz é um producto de differenciação de certas partes da membrana nuclear, de modo que, sempre intimamente ligado a ella, a sua situação topographica em relação á membrana seria, comtudo, variavel, achando-se o filamento ora em relação á sua superficie externa, ora collocado na sua parte mais interna.

Um outro facto sobre o qual desejavamos chamar a attenção é que năo raro se observa no interior de nucleos, cujos filamentos se impregnaram, a existencia de um ou mais corpusculos corados homogeneamente em amarello claro ou em pardo escuro, globulosos, nitidamente delimitados, ora occupando o centro nuclear, ora excentricos (fig. 3). Ese facto da prata reduzida se depositar em um mesmo nucleo contemporaneamente sobre o filamento argentophilo e sobre esses corpusculos, que representam indubitavelmente plasmosomas ou nu= cleolos verdadeiros, constitue uma nova confirmação á opinião professada por HORTEGA e GORRIZ sobre a natureza não nucleinica to filamento argentophilo.

Finalmente, devemo-nos referir a certas formas atypicas muito a aras do filamento perinuclear, observadas nas nossas preparaçóes Nessas formas o filamento apresentava aspectos muito diversos do typo descripto anteriormente. Num dos nucleos, (fig. 4-A), uma das voltas da espiral, que em certo ponto se mostrava muito espessada, se subdividia em tres alsas, que logo se afastavam idivergindo para envolver o nucleo, e das quaes algumas se conservavam muito delgadas e a outra se espessava notavelmen'te. Em um outro nucleo (fig. 4-B), o filamento tendo perdido completamente sua disposição primitiva em espiral, mostrava regiōes irregularmente espessadas para as quaes convergiam ramos argentophilos ora delgados, ora muito espessos, de modio a formar sobre a superficie externa do nucleo uma rêde grosseira de largas malthas e aom os pontos nodaes mais ou menos manifestos.

\section{Filamento perinuclear no myocardio}

Como dissemos ao iniciar esta nota, o methodo urano-argentico de Cajal, no que đdiz respeito á demonstração do filamento perinuclear, não revelou no myocardio a mesma presteza e a mesma segurança nos resultados que nas outras variedades de tecido muscuiar. Com etfeito, fomos obrigados a effectuar muitas tentativas em corações de carneiro. porco e cobaya para só conseguirmos obter am peque- 
no numero de preparados demoinstrativos de myocardio de cobaya e, mesmo nesses preparados, a quantidade de nucleos que apresentavam - apparelho filamentar impregnardo foi ainda muito escassa.

Qual poderia ser a causa das difficuldades assignaladas? A este respeito devemos notar que a impregnação do filamento se operou em regiões onde o tecido cardiaco parecia ter sido ligeiramente dilacerado pelas manipulações technicas preliminares, como si os liquidos reagentes tivessem encontrado nessas soluções de continuidade do tecido condições melhores de penetração. De outro lado, nas fibras estriadas dos musculos voluntarios, onde os nucleos são mais accessiveis, adossados como se acham ao sarcolemma na peripheria dos feixes myofibrillares, a impregnação do filamento é relativamente muito mais facil, como sóe acontecer tambem com as fibro-cellulas, onde, devido talvez a escassez das myofibrillas, os reagentes penetram quasi sem impecilios. Por todas essas razões, suppomos serem as difficuldades mencionadas devidas á posição central quie o nucléo occupa nes segmentos cardiacos, de modo que a espessa massa myofibrillar que os circumda constituiria uma verdadeira baric:ra, collocando-os ao abrigo dos liquidos reagentes.

Entretanto, máo grado essas grandes difficuldades encontradas, nos nucleos aos quaes nos rẹferimos acima, a impregnação se realizou satisfactoriamente, permittindo-nos verificar a existencia em torno delles de um filamento de forma espiral, semelhante ao descripto por HORTEGA nas fibro-cellulas, por GORRIZ e por nós no tecido muscular estriado.

(0) aspecto do fila métío perinuclear no myoicardio. (figs. 5 e 6) obedece mais ou meno so typo classico descripto nas outras modalidades do tecido muscular; parece-nos aṕéna que a sua disposição é aqui um pouco mais sinples, o numero de espiraes variando geralmente entre 3 e. 5 . Em todo caso, reconhecemos à necessidade ide examina um mairt: numero de preparadios para que se possa estabelecer sobre a sua disposição no myocardio uma descripção definitiva.

Muitissimo elegante foi a impregnação do filamento perinuclear nạs cellulas musculares lisás das arterias do coraçâo (fig. 7). Principalmente as arteriolas seccionadas longitudinalmente apresentavam nucleos com - filamento espiral muito homogeneamente impregnado, de aspecto perfeitamente identico aos encontrados por HORTEGA nas fibrocellulas musculares do utero, estomago, intestino, etc.

$\mathrm{O}$ methodo do formol-uranio revelou tambem com alguma constancia no myocardio a presença dos plasmosomas. A differença, porem, do que viramos na musculatura estriada voluntaria, os nucleos cardiacos possuiam um maior numero de nucleolos ve-dadeiros. Geralmente contendo 2 ou 3 relativamcnte grandes, havia nucleos, entretanto. que mostravam até 10 plasmosomas, neste caso muito peque- 
nos, mas sempre com os mesmos caracteres physicos que os plasmosomas da fibra estriada voluntaria.

Como fizemos constar na nos'sa communicação do dia 1 de Julho do corrente anno, fomos surprehendidos em preparados de myocardio de carneiro adulto, executados pelo methodo urano-argentico, com a presença no sarcoplasma que reveste os polos nucleares de um apparelho reticular muito simples, morphologicamente semelhante ao encontrado por DEL RIO HORTEGA nas fibro-cellulas musculares e pelo mesmo A. identificados ao apparelho endocelluiar de Golgi. Devo notar que, no myocardio, o: apparelho reticular interno só foi descriplto até agora por LUNA (1911) (1), cujo trabalho ainda não me foi piossivel consultar.

\section{Ćonclusø̃es}

Dc conjuncto das observações até agora realizadas inferimos logicamente o seguinte:

1. - O filamento perinuclear de HORTEGA-GORRIZ, descipto no nucleo das fibro-cellulas musculares e nos nucleos da fibra muscular estriada, existe tambem no nucleo dos segmentos cardiacos.

2. - E' portanto, um orgão a considerar-se normal provavelmeinte $\mathrm{cm}$ todos os elementos do tecido muscular, isto é, em todos os eleraentos cuịo protoplasma è differenciado para a funcção da contractiiidade.

3. - $\mathrm{O}$ dicto filamento faz parte integrante da membrana nuclear, da qual elle é um producto de differenciação.

1. -- Alein dos argumentos lembrados por HORTEGA e GÓRRIZ, a impregnação argentica simultanea do filamento em questão e dos nucleolos verdadeiros demonstra ainda uma vez ser a substancia que o constitue differente da chromatina nuclear.

Pretendo, porém, continuar as minhas pesquizas em outras differentes formas de tecido musicular, istio é, na membrana myoide dilatadora da pupilla, nas fibro-cellulas musculares de typo lateral das glandulas sudoriparas dos mammiferos, e estender tambem as' minhas observações aos elementos conţracteis lisos e estriados dos invertebrados.

Como resultados dessas novas pesquizas completar-se-ão as presentes conclusões, sendo que algumas serão talvez eventualmente modificadas.

(1) CAJAL - Manual de histologia normal, pag. 453, 7.* edição, Madrid, 1921. 


\section{Explicação das figuras}

(Desemhcs executados pelo A. com o auxilio da camara cla:a de Leitz, object. Bausch \& Lomb $1,9 \mathrm{mms}$. Todas as figuras forem tiradas de preparaçōes obtidas com o methodo urano-argentico de Cajal).

Fig. 1. - Musculo largo abdominal de homem adulto. Nucleo seccionado tangencialmente á face mostrando o filamento espiral perinuclear na sua disposição mais commum.

Fig. 2. - Id. Nucleo com o filamento espiral apparentemente integro, mas cuja alsa central parece estar descollada da membrana nuclear

Fig. 3. - Id. Nacleos cortados tangencialmente ás margens nos quaes houve a impregnação simultanea do apparelho filamentoso e dos nucléolos verdadeiros.

Fig. 4=A e B. - Id. Nucleos envoltos por apparelhos filamentosos atypicos. Em $\mathbf{A}$ não se distingue mais a primitiva disposição em espiral do filamento, observando-se apenas pequenos ramos argentophilos, ou subtis ou muito espessos, irregularmente anastomosados sobre a superficie nuclear. Em B. uma das alsas do filamento a 1 gmenta gredualmente de espessura e afinal se trifurca.

Figs. 5 e 6. - Musculo cardiaco de cobaya. Segmentos myocardicos em cujos nucleos se nota a presença do filamento espiral de HORTEGA-GORRIZ. Na fig. 5 segue-se facilmente todo o trajecto do filamento em torno do nucleo.

Fig. 7. - Seç̧ão longitudinal tangencial de uma arteriola no myocardio de cobaya. Nucleos em bastonete das fibro-cellulas musculares com o filamento argentophilo energicamente impregnado. 


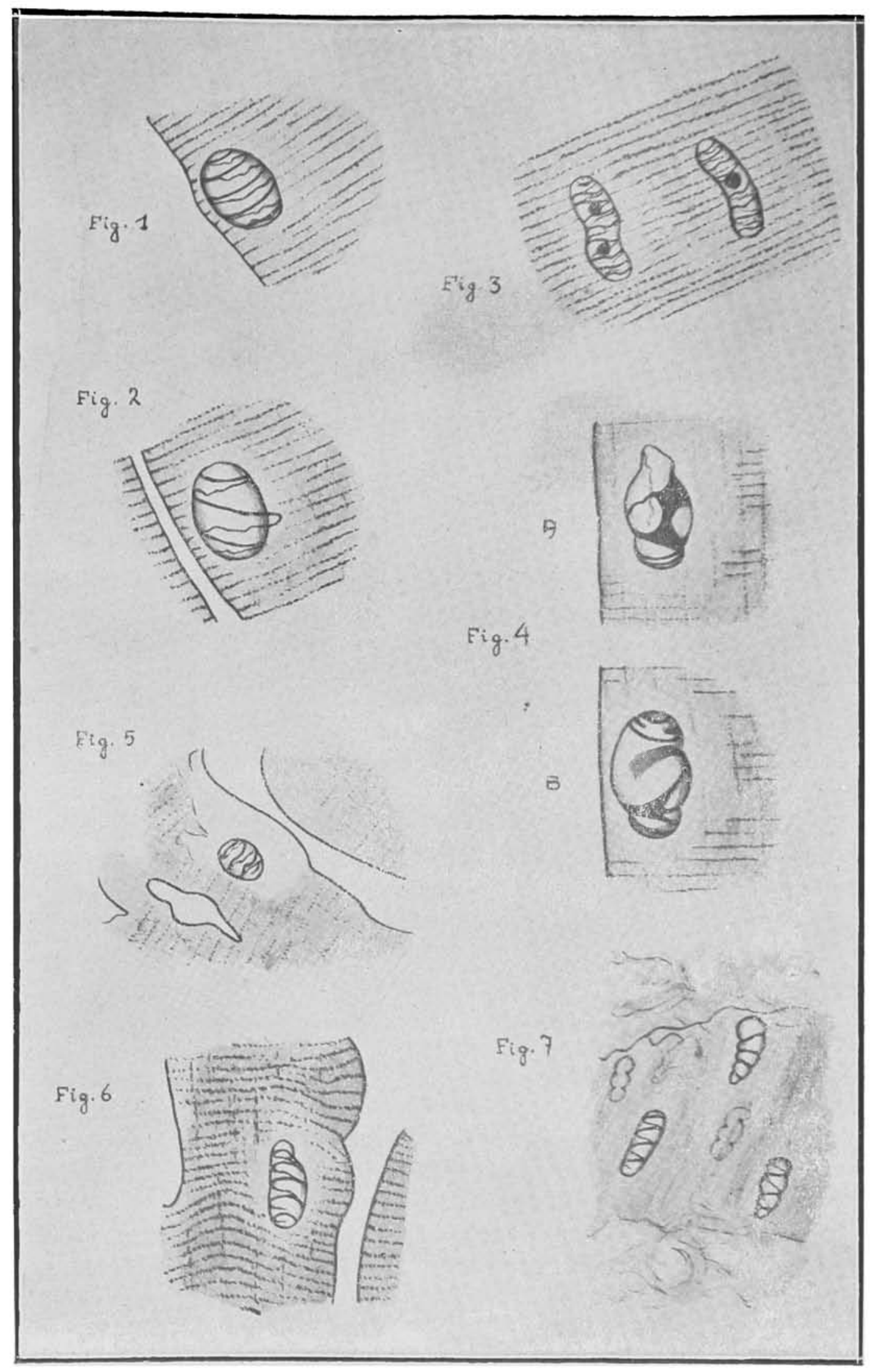

